\title{
TRATAMIENTO ORTODÓNTICO CON DISYUNTOR HYRAX EN PACIENTE DOLICOFACIAL CON AUSENCIAS TÍPICAS
}

\section{ORTHODONTIC TREATMENT WITH HYRAX BREAKER IN DOLICOFACIAL PATIENT WITH TYPICAL ABSENCES}

\author{
Ramos-Montiel Ronald. ${ }^{1 *}$, Valencia-Berrezueta Marcela. ${ }^{2}$ \\ ${ }^{1}$ Od. Esp. Docente Universidad Católica de Cuenca, Docente Posgrado de Ortodoncia Dr. José Apolo Pineda Universidad \\ Estatal de Guayaquil.Ecuador. \\ ${ }^{2}$ Estudiante de Odontología, Universidad Católica de Cuenca.Ecuador \\ *ronald_mtz@hotmail.com
}

\begin{abstract}
Resumen
Se incluye dentro de los hábitos orales a la respiración bucal siendo común en el desarrollo por causas como son: adenoides y las desviaciones del septum nasal, etc. Dentro de la respiración bucal tenemos repercusiones sistémicas, además alteraciones en el desarrollo maxilofacial y mal oclusiones. Desde hace un siglo las investigaciones sobre del desarrollo transversal temprano de los procesos del maxilar superior o la disyunción real de la sutura media palatina, de niños y adolescentes por medio de tornillos intraorales fijos se ha convertido en una importante y exitosa técnica ortopédica y ortodontica. Es por eso que en este caso se trabajó con un disyuntor tipo hyrax en un paciente con Ausencias Típicas, respiración bucal, septum nasal desviado, apiñamiento dental y mal oclusión.
\end{abstract}

Palabras clave: Disyunción, ausencias, tabique maxilar.

\begin{abstract}
Talking about oral habits, include oral breathing, which is frequently because of many factors like: adenoids and a bad position of the nasal septum, etc. Oral breathing can start systemic complications, plus some problems in maxillofacial development and malocclusion. For a century research on early development of the transverse processes of the upper jaw or the actual disjunction of the palatal suture half of children and adolescents through intraoral fixed screws it has become an important and successful orthopedic and orthodontic technique. That is why this case it worked with a type hyrax breaker in a patient with petit mal seizures, mouth breathing, nasal septum diverted, dental crowding and malocclusion.
\end{abstract}

Key words: Expansion, absences, Maxillary septum.

\section{INTRODUCCIÓN}

Se aconseja comenzar a actuar ortopédicamente en pacientes que presenten limitantes en el funcionamiento estomatognático en edades tempranas debido a que es en el crecimiento donde se puede corregir o prevenir discrepancias maxilo-mandibulares, apiñamiento dental; los cuales de no ser tratados oportunamente puede terminar en un tratamiento quirúrgico no deseado. La expansión rápida del maxilar es un método fácil de realizar y de bajo costo por medio de un disyuntor aparato que aumenta la dimensión vertical y transversal del maxilar y de la mandíbula, por ende logra aumentar el espacio de la arcada, el mismo que podría ser usado para evitar extracciones dentales o cirugía posterior ${ }^{1,7,8}$

En la actualidad se han descrito diversas técnicas ortodónticas aplicadas en pacientes con maloclusiones trans- versales y una es la expansión rápida del maxilar que fue un método descrito por Angle en 1860 diseñando el primer aparato que mediante fuerzas ortopédicas llegaba a separar la sutura media palatina ${ }^{1,2}$ y fue Praff que lo usó en pacientes mayores de 16 años. Hoy en día es utilizado en la práctica diaria del ortodoncista. ${ }^{2-4}$

La expansión rápida del maxilar se utiliza en pacientes con inhibición del crecimiento del maxilar superior, mordida cruzada bilateral, hendiduras labio palatinas, preparación para cirugía ortognática u ortopedia funcional, no se recomienda su aplicación en pacientes que no cooperan con el tratamiento, para corregir un diente cruzado, en asimetría maxilar o mandibular o en adultos con graves interferencias esqueletales, ya sean anteroposteriores, transversales o verticales. ${ }^{5,6}$ 


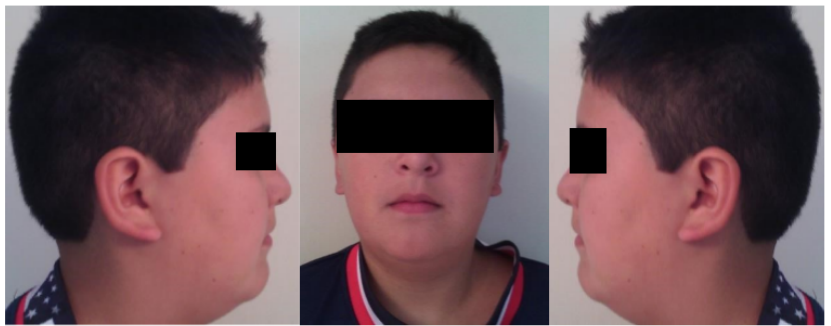

Fig. 1. Fotografías Iniciales; Fotos Extra orales A. Perfil derecho B. Frontal C. Perfil Izquierdo

A B

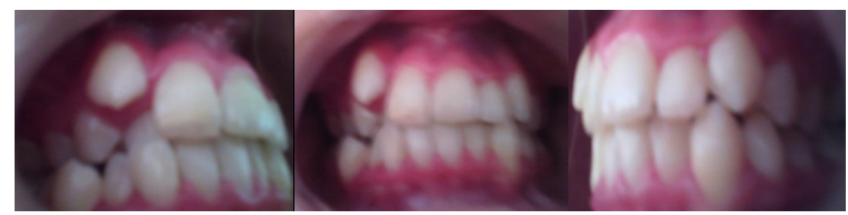

Fig. 2. Fotografías Intra orales

Las ausencias típicas son tipos de epilepsias momentáneas sin causa aparente que se caracteriza por episodios de tiempo cortos, en este tipo de patología el cerebro no es bien oxigenado ${ }^{9,10}$ y paralelamente a los problemas dentales que presente un paciente expandiendo el maxilar podríamos lograr no solo tener espacio en la arcada dental además arreglar el septum desviado y por ende más oxigenación al cerebro logrando dar una mejor calidad de vida a nuestro paciente.

\section{PRESENTACIÓN DEL CASO}

\subsection{CASO CLÍNICO EXÁMEN EXTRAORAL DE INICIO}

Paciente de sexo masculino de 13 años de edad presenta Ausencias Típicas, asimetría facial, sus tercios se encuentran alterados, siendo mayor el inferior, seguido del medio y el de menos diámetro el superior, presenta desviación de la línea media. (Figura 1)

Clínicamente se observa pieza 1.3 ectópico; 1.2 retruída (Figura 2A), Mordida profunda, desviación de la línea media superior e inferior, apiñamiento inferior y supe22rior (Figura 2B). Se observa además lo característico del Biotipo Dolicofacial o leptoprosopo, las piezas dentales tienen forma alargada con unos vértices cuspídeos alargados que mejoran notoriamente los movimientos de lateralidad de la oclusión. Paladar profundo (Figura 3A), Apiñamiento inferior (Figura 3B)

\subsection{ESTUDIO RADIOGRÁFICO CEFALOMÉTRICO LA- TERAL}

Se realizó un estudio Cefalométrico de Björk Jarabak, además de los ángulos de clase esqueletal del análisis de

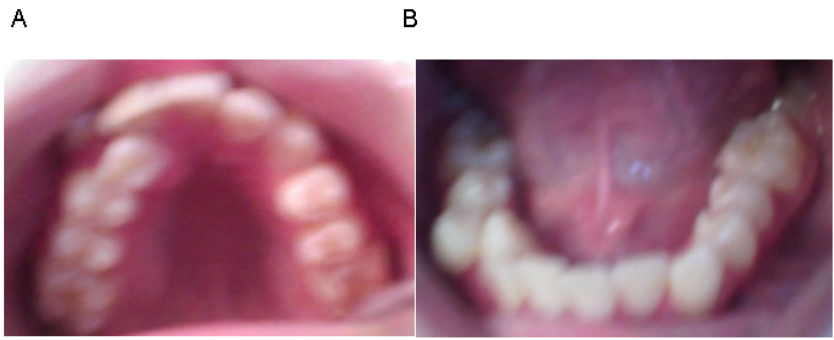

Fig. 3. Fotogarfías Intraorales
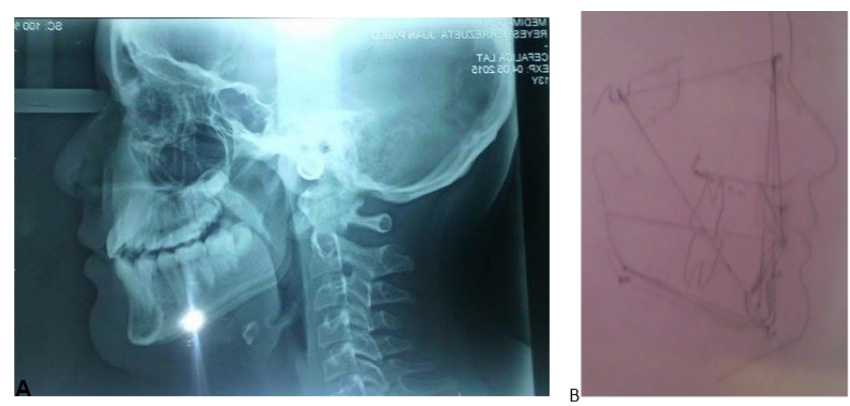

Fig. 4. Radiografía Lateral del cráneo

Steiner

\subsection{DIAGNÓSTICO RADIOGRÁFICO}

- Clase esqueletal III porque presenta crecimiento vertical

- Mordida profunda. Incisivos superiores e inferiores proinclinados.

- Perfil Facial: Convexo

- Biotipo: Dolicofacial

- Diagnóstico Radiográfico Paciente presenta simetría en los puntos radiográficos analizados, desviación del septum nasal, las fosas nasal derecha se encuentra ligeramente descubierta para el paso del aire mientras que la izquierda se encuentra completamente tapada.

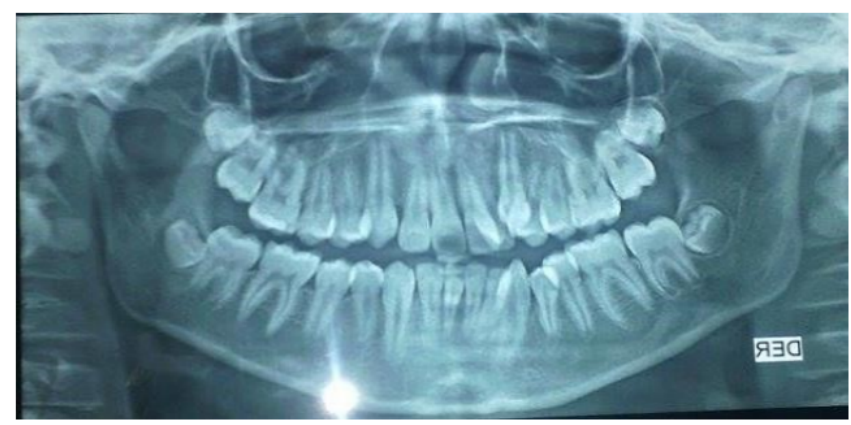

Fig. 5. Radiografía Panorámica 


\begin{tabular}{|c|c|c|c|}
\hline $\begin{array}{l}\text { PUNTOS } \\
\text { CEFALOMÉTRICOS }\end{array}$ & $\begin{array}{l}\text { MEDIDAS DEL } \\
\text { PACIENTE }\end{array}$ & $\overline{\text { NORMA }}$ & DIAGNOSTICO \\
\hline $\begin{array}{c}\text { Base Craneal } \\
\text { Posterior }\end{array}$ & $42 \mathrm{~mm}$ & $31 \mathrm{~mm}+1-4$ & Crecimiento Vertical \\
\hline Base Craneal Anterior & $70 \mathrm{~mm}$ & $71+1-3$ & Normal \\
\hline Altura Facial Posterior & $82 \mathrm{~mm}$ & $70 \mathrm{~mm}$ a $85 \mathrm{~mm}$ & Normal \\
\hline Altura Facial Anterior & $120 \mathrm{~mm}$ & $105-120 \mathrm{~mm}$ & Normal \\
\hline Go-Me & $65 \mathrm{~mm}$ & $71+/-5$ & Posible Clase III \\
\hline N-Me a S-Go & 68.33 & & Dolicofacial \\
\hline Altura de la Rama & $44 \mathrm{~mm}$ & $44+/-5 \mathrm{~mm}$ & Normal \\
\hline N-S-Ar & $115^{\circ}$ & $123^{\circ}+3-5$ & $\begin{array}{l}\text { Mordida Abierta } \\
\text { Profunda }\end{array}$ \\
\hline Ar-Go-Me & $134^{\circ}$ & $130^{\circ}+/-7$ & Normal \\
\hline $\mathrm{Ar}-\mathrm{Go}-\mathrm{N}$ & $54^{\circ}$ & $52^{\circ}$ a $55^{\circ}$ & Normal \\
\hline Ar-Me-Na & $77^{\circ}$ & $70^{\circ}$ a $75^{\circ}$ & Mordida Profunda \\
\hline SNA & $77^{\circ}$ & $82^{\circ}$ & Retruída \\
\hline SNB & $78^{\circ}$ & $80^{\circ}+1-3^{\circ}$ & Normal \\
\hline ANB & $-1^{\circ}$ & $4^{\circ}+1-2^{\circ}$ & Clase III \\
\hline Ángulo 1-NA & $22^{\circ}$ & $22^{\circ}$ & Normal \\
\hline Distancia 1-NA & $4 \mathrm{~mm}$ & $4 \mathrm{~mm}$ & Normal \\
\hline Ángulo 1-NB & $30^{\circ}$ & $25^{\circ}$ & Proinclinado \\
\hline Distan cia $1 \mathrm{NB}$ & $6 \mathrm{~mm}$ & $4 \mathrm{~mm}$ & Proinclinado \\
\hline Plano Oclusal a SN & $14^{\circ}$ & $14^{\circ}+1-5$ & Normal \\
\hline Ángulo MM & $14^{\circ}$ & $27^{\circ}$ & Extracciones \\
\hline
\end{tabular}

Tabla 1. Medidas Cafalométricas

\begin{tabular}{llll}
\hline $\begin{array}{l}\text { PUNTOS A } \\
\text { EVALUAR }\end{array}$ & DERECHO & IZQUIERDO & DIFERENCIA \\
\hline Co-Go & & & \\
Go-Si & $52 \mathrm{~mm}$ & $52 \mathrm{~mm}$ & $0 \mathrm{~mm}$ \\
R1-R2 & $85 \mathrm{~mm}$ & $85 \mathrm{~mm}$ & $0 \mathrm{~mm}$ \\
\hline
\end{tabular}

Tabla 2. Medidas a evaluar 
A
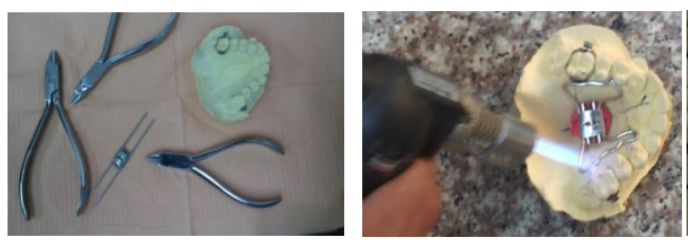

Fig. 6. Elaboración del Disyuntor

\subsection{DIAGNÓSTICO RADIOGRÁFICO}

Paciente de sexo masculino presenta ausencias típicas, en cavidad bucal presenta mal oclusión, apiñamiento, canino ectópico, la línea media superior no coincide con la inferior, mordida profunda, incisivos superiores e inferiores proinclinados, asimetría en su rostro, perfil facial convexo, Dolicofacial presenta crecimiento vertical, desviación del septum nasal, las fosas nasal derecha se encuentra ligeramente descubierta para el paso del aire mientras que la izquierda se encuentra completamente tapada.

\subsection{PLAN DE TRATAMIENTO}

- Crear espacio en la arcada dental para aliviar el apiñamiento.

- Crear espacio para la posterior ortodoncia.

- Evitar futuras extracciones.

- Mejorar la forma del paladar, corrigiendo el hábito de respirador bucal a nasal mediante la apertura de las fosas nasales.

- Mejorar la desviación del septum nasal.

\subsection{ETÁPAS DEL TRATAMIENTO}

Instrumental para la elaboración del Hyrax, (figura $6^{\text {a }} \mathrm{Y}$ 6B), Espatulado del ionómero elegido para el cementado del tornillo de Hyrax. (Figura 6C).

\subsection{FINAL DEL TRATAMIENTO EXAMEN}

Se ha logrado llevar la mordida a posición bis a bis, mayor espacio interdental, y se logró abrir el diastema a nivel de los incisivos superiores. (Figura 10 A-B-C)

El tornillo está abierto en su totalidad, se observa mejora en la forma del paladar (Figura $10 \mathrm{~A}$ ), Se observa más espacio en la arcada inferior para producir movimientos ortodónticos posteriores. (Figura $10 \mathrm{~B}$ )

El tratamiento tuvo una duración de 25 días en los cuales se activó el tornillo dos cuartos de vuelta en la mañana y dos cuartos de vuelta en la tarde, se insistió en la higiene bucal del paciente y una alimentación blanda y balanceada a nivel dental se logró crear espacio interdental mediante el diastema a nivel incisal, se logró expandir el maxilar superior logrando mejorar la forma del paladar, a nivel radiográfico se observa como las fosas nasales se abrieron especialmente a

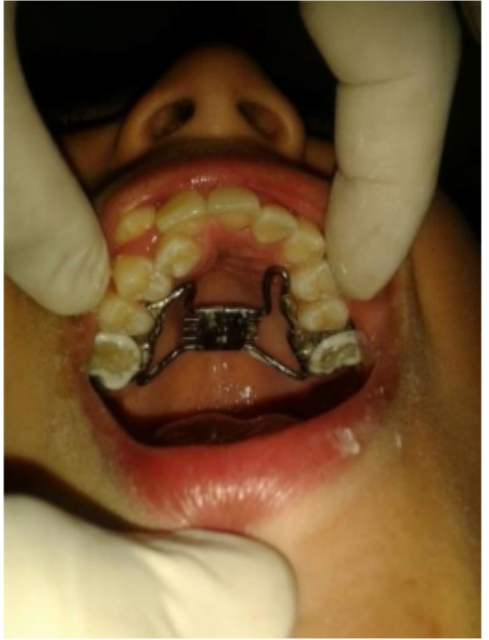

Fig. 7. Cementado en la boca del paciente

A

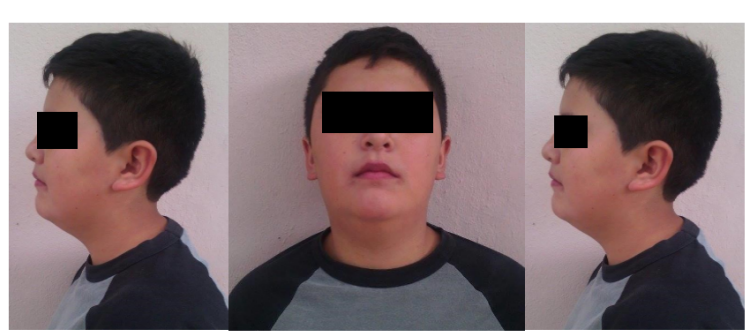

Fig. 8. Fotografías Finales; Fotos Extra oralesA. Lateral Derecha B. Frontal C. Lateral Izquierda

nivel derecho se observa despejada a nivel izquierdo tenemos ligeramente abierto, y el septum ligeramente a cambiado.

\section{RESULTADOS}

- Diastema a nivel de los incisivos centrales superiores.

- Se mejoró la forma del paladar.

- Se abrieron las fosas nasales, mejorando la respiración del paciente por ende mayor cantidad de oxígeno al cerebro y mejorando su patología que son las ausencias.

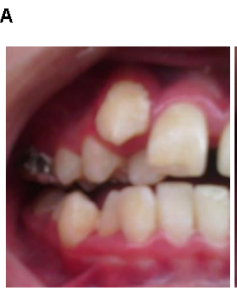
B C

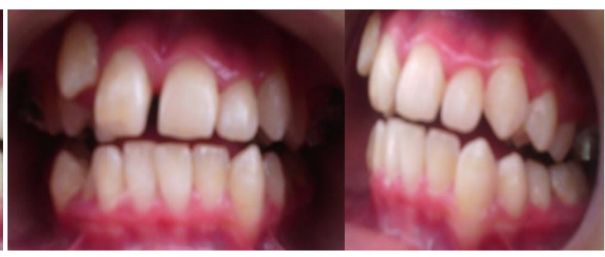

Fig. 9. Fotos Intraorales segundo momento 


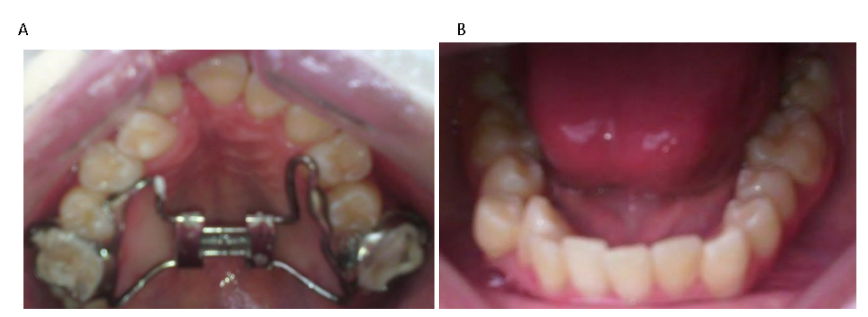

Fig. 10. Fotos Intraorales tercer momento

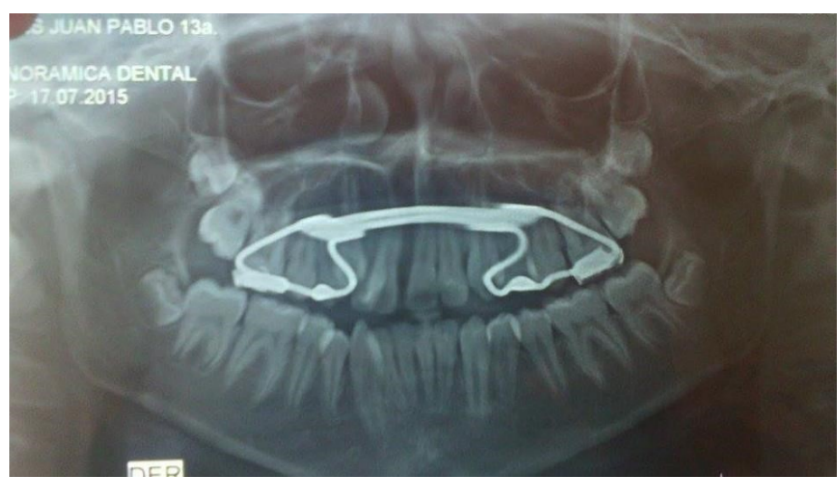

Fig. 11. Radiografía panorámica final

\section{DISCUSIÓN}

Los disyuntores tipo Hyrax nos facilitan corregir problemas transversales del maxilar superior asociadas a defectos de la base esquelética y relacionada con maloclusiones de clases tipo I, II y III. Los disyuntores tipo Hyrax son muy fáciles de limpiar y cómodos en relación a los demás tipos de disyuntores además que el resultado es rápido, en el paciente en estudio se logró separar la sutura media palatina con una mínima inclinación de molares y premolares llegando a una mordida bis a bis, aumentando la longitud de la arcada dental superior y su alineación logrando así relación oclusal demostrando la efectividad del tratamiento con el uso de disyuntores, cabe mencionar que durante la elaboración de este artículo no se encontraba en la etapa final del tratamiento, sin embargo se lograron en gran parte los objetivos trazados. ${ }^{6} \mathrm{La}$ disyunción con Hyrax se realiza como tratamiento ortopédico temprano previo a la utilización de ortodoncia. ${ }^{6}$

\section{CONCLUSIÓN}

La disyunción rápida del maxilar es una alternativa adecuada siempre y cuando se realice un diagnóstico previo, tener en cuenta el biotipo facial del paciente, la edad y la disposición del paciente y la familia de colaborar con el tratamiento. En nuestro caso en particular se cumplieron los objetivos trazados como expandir el maxilar, corregir el hábito de respiración bucal, corregir el septum desviado. Además logramos una mejor apariencia para nuestro paciente, consiguiendo el espacio adecuado para la posterior ortodoncia.

\section{RECOMENDACIONES}

En este tipo de pacientes es necesario realizar un correcto diagnóstico, y realizar primero la disyunción rápida del maxilar para producir espacio esqueletal y dental para posteriores tratamientos ortodónticos, evitando así futuras exodoncias y cirugías. Control y evolución de pacientes con tipos de epilepsias y mal oclusión dental.

\section{Referencias}

1 Mata J., Zambrano F., Quirós O., Farias, M., Rondón, S., Lerner, H Expansión Rápida de Maxilar en Maloclusiones Transversales. Revisión Bibliográfica.Rev. Latinoamericana de Ortodoncia y Odontopediatria 2009.Disponible en

2 Peña M. Cambios dentales en pacientes de 8-14 años de edad con compresión maxilar, utilizando expansión rápida maxilar con hyrax, universidad autónoma de Nuevo León Facultad de Odontología, 2014.

3 Gómez R, Arias M, López Y, González D3 Disyunción maxilar rápida con tornillo Hyrax modificado.

4 Adkins, MD. Arch perimeters dianges on rapid palatal expansion. Am J Orthod Dentofac Orthop 97:194, 1986.

5 Silva F, Rivas G Disyunción palatina, tratamiento ortodóncico temprano: Reporte de Caso.

6 Reyes M, Comas R, Martínez M, Mock P, Expanción rápida del maxilar con el tornillo hyrax en un adolecente. Rev. Scielo (2015) [Internet] (Citado en: 12/06/2015) 110.Documento disponible

7 Machado R, Bastidas M, Arias E, Quirós O. Disyunción maxilar con la utilización del expansor tipo Hyrax en pacientes con labio y paladar hendidos. Revisión de la Literatura. Rev Latin Ortod Odontol. 2012 [citado 13 Jun 2015].

8 Chacón Dávila MM. Efectos inmediatos dentales y esqueléticos de la expansión rápida del maxilar con un disyuntor a dos bandas modificado. Tesis doctoral. Universidad de Sevilla, 2014 [citado 2 Ago 2014].

9 Bestene J, Introducción a la clínica, Centro Editorial Javeriano, Pág. 312

10 Villarejo F.Tratamiento a la Epilepsia, Ediciones Días de Santos S.A 1998 Pág. 60 
